Relations industrielles

Industrial Relations

\title{
Les cadres et la révolution informatique, l'adaptation nécessaire, par Arnold Kaufmann, Entreprise Moderne d'Édition, Paris, 1968, 144 pages.
}

\section{André Petit}

\section{Volume 24, numéro 4, 1969}

URI : https://id.erudit.org/iderudit/028090ar

DOI : https://doi.org/10.7202/028090ar

Aller au sommaire du numéro

Éditeur(s)

Département des relations industrielles de l'Université Laval

ISSN

0034-379X (imprimé)

1703-8138 (numérique)

Découvrir la revue

Citer ce compte rendu

Petit, A. (1969). Compte rendu de [Les cadres et la révolution informatique, l'adaptation nécessaire, par Arnold Kaufmann, Entreprise Moderne d'Édition, Paris, 1968, 144 pages.] Relations industrielles / Industrial Relations, 24(4),

814-814. https://doi.org/10.7202/028090ar

Tous droits réservés @ Département des relations industrielles de l'Université Laval, 1969
Ce document est protégé par la loi sur le droit d'auteur. L’utilisation des services d’Érudit (y compris la reproduction) est assujettie à sa politique d'utilisation que vous pouvez consulter en ligne.

https://apropos.erudit.org/fr/usagers/politique-dutilisation/ 
model of organizational growth, with a different approach than those of Schroeder, Haire, Dale and Buchele.

Original presentation, good methodology, extensive bibliography, some innovation, all this makes the Filley-House book an excellent text for anyone interested in recent developments in administrative and behavioral sciences.

\section{J. LADOUCEUR}

Lexique de la psychologie, par Arlette et Roger MUCCHIELLI, Entreprise Moderne d'édition, Editions Sociales Françaises, Paris, 1969, 188 pp.

Lexique des sciences sociales, par Arlette et Roger MUCCHIELLI, Entreprise Moderne d'Edition, Editions Sociales Françaises, Paris, 1969, 196 pp.

Avec le vocabulaire technique que doivent nécessairement utiliser ceux qui veulent faire un travail scientifique, il devient de plus en plus difficile aux non-spécialistes de comprendre les études présentées au public sous forme d'articles ou d'ouvrages. Le domaine des sciences de l'homme est particulièrement peuplé de mots nouveaux ou de mots anciens pris dans une acception particulière.

Le Lexique de la psychologie contient les principaux concepts de la psychophysiologie, de la psychopathologie, de la psychanalyse, des psychothérapies, de la psychopédagogie et de la psychologie des groupes. Le Lexique des sciences sociales est consacré à la psychologie sociale, à la dynamique des groupes, à l'ergonomie, à la sociologie du travail et aux théories des communications et de l'information.

Ces deux ouvrages viennent aider les étudiants, les profanes et même les praticiens. Ils sont loin d'être complets et on pourra discuter l'une ou l'autre des définitions ou des explications que les auteurs proposent. II n'en reste pas moins que sous une présentation simple et d'une lecture facile, ils sont très utiles.
Les cadres et la révolution informatique, l'adaptation nécessaire, par Arnold Kaufmann, Entreprise moderne d'édition, Paris, 1968, 144 pages.

Arnold Kaufmann nous présente ici un sujet fleuve. Son intention est de motiver le lecteur et de l'amener à réfléchir sur la nécessaire éducation permanente à l'âge de l'ordinateur. Il nous présente ce qu'il appelle un combat contre certains préjugés et contre de nombreuses habitudes assez anciennes. S'adressant au cadre, il veut le persuader que son rôle dans la nouvelle société, sera justement de créer, encore plus que les autres, de manière que ces autres aient envie de se surmonter à leur tour. Ses considérations qui s'étendent sur un peu plus d'une centaine de pages, sont recoupées en quelque vingt-six chapitres.

L'auteur traite évidemment des cadres, surtout des cadres français. A partir d'études psycho-sociologiques, il en donne les caractéristiques principales et décrit particulièrement leurs attitudes face à l'ère des ordinateurs. La technologie de l'ordinateur et l'adaptation nécessaire de l'homme à ce nouvel instrument sont ensuite les préoccupations qui donnent lieu au plus grand nombre de chapitres. L'adaptation en termes de connaissances se traduit, entre autres, par le chapitre 8: «De nouvelles et indispensables connaissances pour les cadres $»$. L'importance des mathématiques modernes, la spécialisation et la qualification adaptable, l'imprégnation culturelle de même que l'intensification du dialogue homme-homme sont les sujets de chapitres subséquents. Des considérations plus globales sur la place de l'homme dans cette nouvelle société se traduisent par des chapitres comme: «Capitalisme et socialisme à l'ère des ordinateurs», «Les banques de données ou la liberté trahie », «La civilisation promotionnelle ». M. Kaufmann termine par quelques remarques supplémentaires sur le rôle des cadres et leur éducation permanente.

Cet ouvrage peut reconcilier avec l'informatique les cadres qui seraient peu ou pas convaincus. Nous souhaitons, avec l'auteur, qu'il puisse atteindre son but. 